\title{
Environmentally Benign Mortar-Pestle-Induced Acylation and $O$-Alkylation of Aromatic and Heteroaromatic Compounds under Solvent-Free Micellar Conditions and Computation of Their Drug Likeliness Properties
}

\author{
Kancharla Rajendar Reddy, Kamatala Chinna Rajanna, Kusampally Uppalaiah, \\ Mukka Satish Kumar, and Marri Venkateshwarlu
}

Department of Chemistry, Osmania University, Hyderabad 500 007, India

Correspondence should be addressed to Kamatala Chinna Rajanna, kcrajannaou@yahoo.com

Received 12 September 2012; Accepted 10 November 2012

Academic Editor: Paul Watts

Copyright (C) 2012 Kancharla Rajendar Reddy et al. This is an open access article distributed under the Creative Commons Attribution License, which permits unrestricted use, distribution, and reproduction in any medium, provided the original work is properly cited.

Environmentally benign mortar-pestle-induced practical methods have been developed for the acylation and $O$-alkylation of aromatic and heteroaromatic compounds under solventfree micellar conditions, which were found to efficiently afford moderate to excellent yields of products.

\section{Introduction}

The diverse nature of chemical universe requires various green strategic pathways in our quest towards attaining sustainability. The emerging area of green chemistry envisages minimum hazard as the performance criteria while designing new chemical processes. One of the thrust areas for achieving this target is to explore alternative reaction conditions and reaction media to accomplish the desired chemical transformations with minimized byproducts or waste as well as eliminating the use of conventional organic solvents, wherever possible. Consequently, several newer strategies have appeared such as solvent-free reactions (grinding), multicomponent reactions under solvent-free conditions could enhance their efficiency from an economic as well as an ecological point of view the solid-state organic reactions is gaining significance both from the mechanistic and synthetic point of view [1-3]. Number of articles are available reporting solid-state reactions by grinding such as, Grignard reaction [4], Reformatsky reaction [5], Aldol condensation [6], Dickhmann condensation [7], phenol coupling reaction [8], reduction reaction [9], Wittig reaction
[10], Grignard and McMurry reaction [11], and Synthesis of Polyhydroquinolines [12]. In recent past, our research group is actively involved in the exploration of in the exploration of nonconventional methods for nitration, bromination, acetylation, and benzylation reactions in the direction of achieving greenery conditions [13-16]. Most of these griding reactions are carried out at room temperature, absolutely solvent-free and use only a mortar and pestle and also economical and green procedures. Therefore, we focus on developing the novel procedure involving a solid-state reaction performed by grinding.

Alkylated and acylated aromatic and heteroaromatic compounds are significant precursors in drug intermediates and industrial uses [17]. Specifically naphthalene derivatives such as naphthol ethers and acylated naphthol ethers have been identified as one of the best ranges of potent antimicrobials effective against wide range of human pathogens. A perusal of literature indicated that Williamson synthesis is probably one of the most common classical methods being used for the preparation of symmetrical and unsymmetrical ethers [18-24]. Recent publications of 


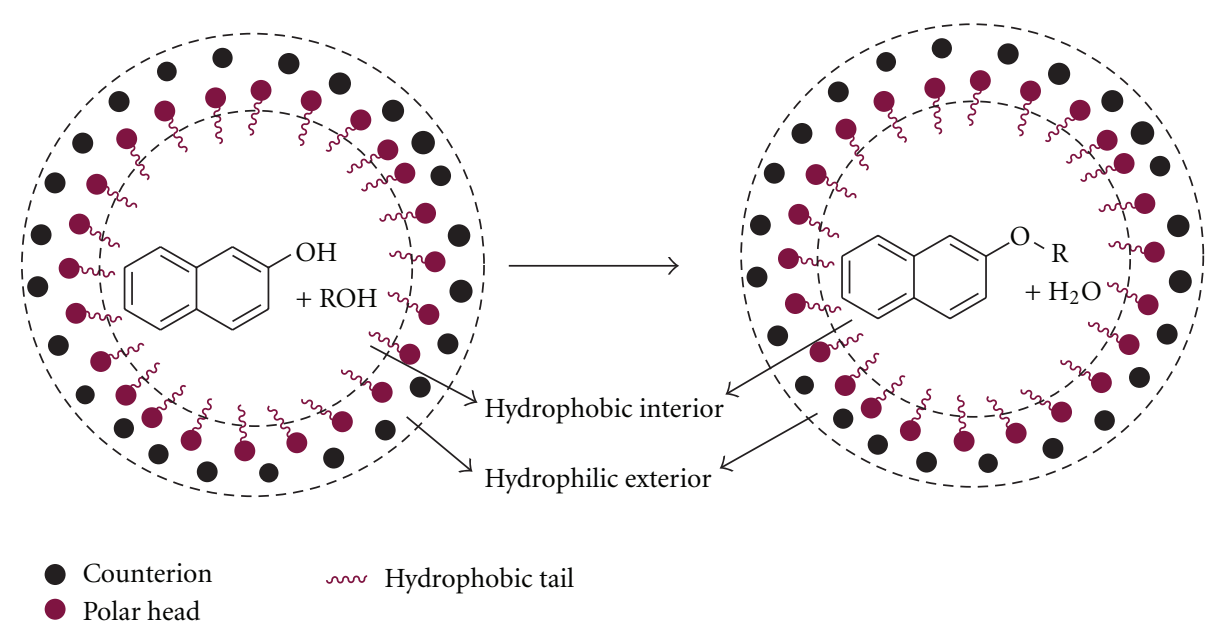

FIGURE 1: Schematic diagram representing the role of micelles in the reaction.

Swamy et al. [25], Debabrata and coworkers [26, 27], provide excellent bibliography and information on the synthesis of aryl, heteroaryl ethers derived from various roots.

The Friedel-Crafts reaction has a long history in organic synthesis for electrophilic aromatic substitution reactions such as alkylations and acylations. To date these reactions are of great importance for the synthesis of aromatic carbonyl compounds [28]. Aromatic ketones can also be prepared by the reaction of carboxylic acids with aromatic hydrocarbons catalyzed by Nafion-H [29]. A significant number of Lewis acid catalysts $\left(\mathrm{AlCl}_{3}, \mathrm{FeCl}_{3}, \mathrm{SnCl}_{4}\right.$, and $\left.\mathrm{BF}_{3} \bullet \mathrm{OEt}_{2}\right)$ have been shown to be very successful for the acylation of aromatic substrates with acid chlorides or anhydrides, while the reaction is usually carried out using a stoichiometric amount of $\mathrm{AlCl}_{3}$, reactive aromatic compounds are known to undergo acylation in the presence of a catalytic amount of Lewis acid. Recently, several effective catalyst systems for the acylation of anisole and its derivatives have also been developed [30-34]. A perusal of literature indicated that surfactants have been used to promote a variety of synthetic organic reactions [35-37]. Our preliminary studies in this direction ended up with fruitful results when we have performed the title reactions in presence of micelle forming anionic (SDS), cationic (CTAB, CTAC), and nonionic (Tx-100) surfactants. This study is aimed at developing "greener synthetic protocols" for etherification and acylation reactions under solvent-free conditions, as encouraged by the "Green Chemistry strategies" of Paul Anastas and Warner [38]. We have used mortar-pestle grinding technique in this study because it is economically cheap and safer method and yet is known to good yields of products in the synthesis of several organic compounds [1-3]. These reactions underwent dramatic rate accelerations under these conditions. We were also successful to develop solvent-free synthetic methods in the above protocols by replacing acids with a variety of micelle forming surfactants such as sodium dodecyl sulphate (SDS), cetyl trimethyl ammonium bromide (CTAB), cetyl trimethyl ammonium chloride (CTAC), and Triton-X 100.

\section{Results and Discussion}

Synthesis of naphthol ethers under Williamson's conditions (refluxing in sulfuric acid and organic solvent) required several hours $(\geq 20 \mathrm{~h})$ at relatively high temperature. Even though there are some reports to modify the drastic conditions of these reactions, many of them exhibited long reaction times, accumulation of unwanted byproducts, which ultimately involved tedious work-up procedures. Encouraged by this aspect, we have conducted $O$-alkylation of aromatic and heteroaromatic alcohol reaction (Scheme 1) in micellar media under acid-free conditions.

However, for comparison, we have also conducted etherification of $\beta$-naphthol reactions under Williamson's classical conditions in acidic media. Results obtained under acidic and acid-free micellar conditions are compiled in Table 1 and $O$-alkylation of hydroxy pyridines in Table 2 , and the reaction time versus conversion of aromatic/heteroaromatic alcohols using micelles as a catalyst were reported in Table 3, which clearly indicate highly significant rate accelerations followed by very good yield of end products. Catalytic activity of different micelles is in the order: CTAB $>$ SDS $>$ Tx-100. It is believed that micelles themselves act as microreactors. By introducing a surfactant (CTAB), dehydration was successfully achieved. The catalytic effect of the micellar solution of CTAB may be attributed to the hydrophobic nature of organic substrates. Formation of emulsion droplets takes place in water in the presence of surfactant and substrate molecules. It is suggested that most of the organic substrates are concentrated in these spherical droplets, which act as a hydrophobic reaction sites and results in an increase in the effective concentration of the organic reactants, which might increase the reaction rate via a concentration effect. In micellar solution, organic substrates are pushed away from water molecules towards the hydrophobic core of micelle droplets thus inducing efficient collisions between organic substrates which eventually enhance the reaction rates. The hydrophobic interior of the micelles swiftly excludes the water molecules generated during the reaction, thus shifting the equilibrium towards the desired product that ultimately 

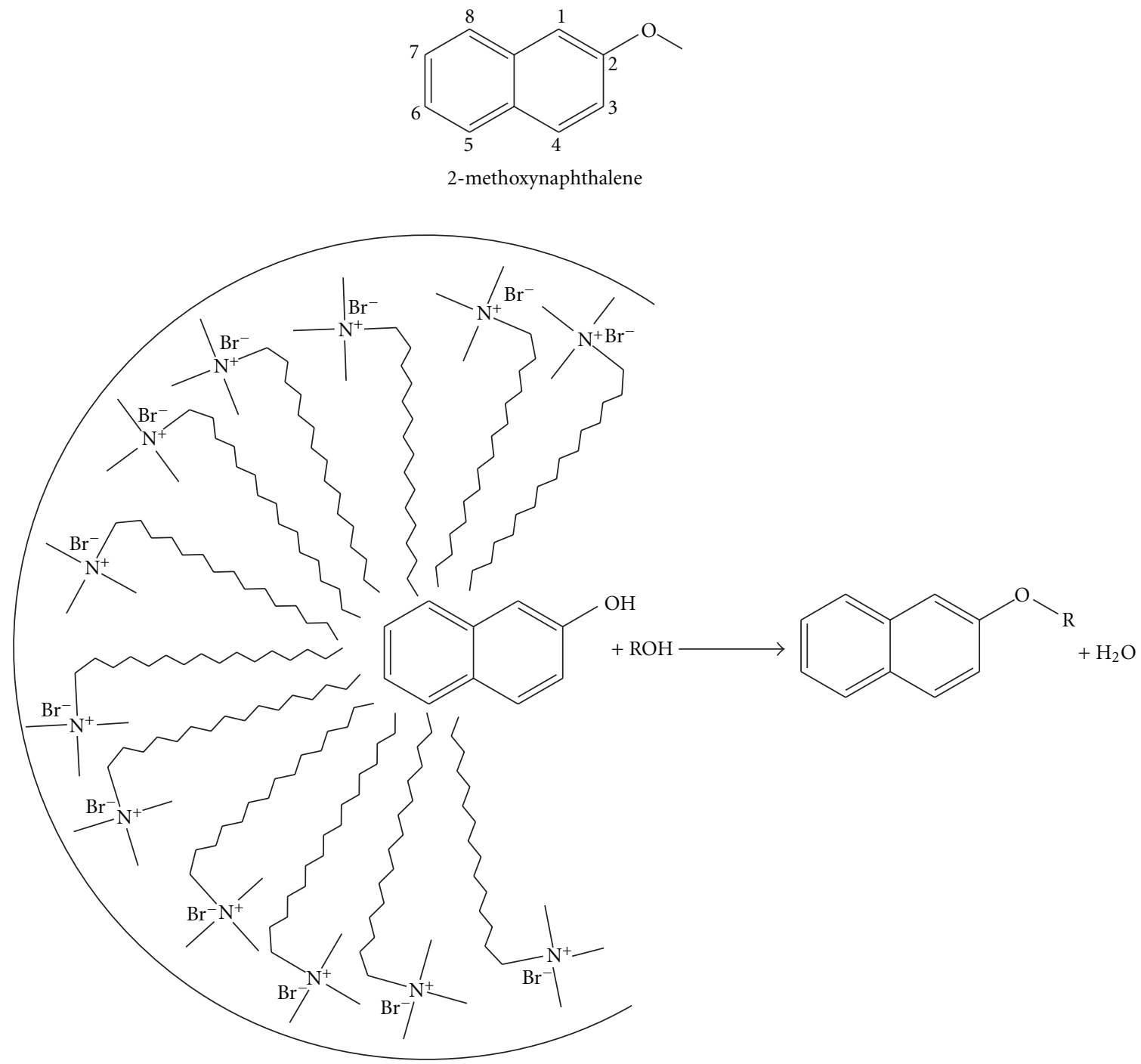

FIGURE 2: Schematic diagram representing the role of CTAB in the reaction.

$$
\begin{array}{cl}
\mathrm{Ar}-\mathrm{OH} \longrightarrow \mathrm{ROH} / \text { micelles } \\
\stackrel{\mathrm{Grinding} \text { conditions }}{\mathrm{Ar}=\text { aromatic/heteroaromatic }} \\
\mathrm{R}=\mathrm{CH}_{3}, \mathrm{C}_{2} \mathrm{H}_{5}, \mathrm{C}_{3} \mathrm{H}_{7} ; \\
\text { Micelles }=\mathrm{CTAB}, \mathrm{SDS}, \mathrm{TX}-100
\end{array}
$$

Scheme 1: $O$-alkylation of Aromatic/heteroaromatic compounds in micellar media.

leads to an increase in the reaction yield $[39,40]$. This explanation is schematically represented in Figures 1 and 2.

The Friedel-Crafts acylation of 2-methoxy naphthalene in the presence of the electron-donating group $(\mathrm{OMe})$ must be taken into account in order to figure out the reaction. Presence of the electron-donating group activates the 1, 6, and 8 positions of the naphthalene ring.
The 1-position is more active than the other two positions and the 6-position is more stable than the other two positions, so acylation of 2-methoxynaphthalene generally occurs at this kinetically favoured 1-position at low temperatures and at the thermodynamically favoured 6-position at high temperatures. At high temperatures, in the reaction mixture, migration of the acyl group from 1- to 6-position which is named as transacylation and protiodeacylation of the acyl group at the 1-position of 1-acyl-2-methoxy naphthalene are possible. These two reactions may result in the formation of the thermodynamically favoured product which is 6-acyl-2-methoxynaphthalene. Steric hindrance to acylation is reported in the order $1>8>6$-position (Das, 2000). Therefore, the isomerisation of the sterically hindered ketone 1-acyl-2-methoxynaphthalene to sterically less hindered isomers like 6-acyl-2-methoxy naphthalene and 8-acyl-2-methoxynaphthalene will be favoured.

Catalytic reaction of 1-halo-2-methoxy naphthalene $(2 \mathrm{mmol})$ was treated with acylchloride $(2 \mathrm{mmol})$ in the 
TAвLE 1: Mortar-pestle-induced $O$-alkylation of $\beta$-naphthols under solvent-free conditions.

\begin{tabular}{|c|c|c|c|c|c|c|}
\hline \multicolumn{2}{|l|}{ Reactants } & \multirow{2}{*}{$\begin{array}{c}\mathrm{H}_{2} \mathrm{SO}_{4} \\
(5 \mathrm{hr}) \\
\% \text { yield }\end{array}$} & \multirow{2}{*}{$\begin{array}{c}\text { CTAB } \\
\text { (2 hr) } \\
\text { \% yield }\end{array}$} & \multirow{2}{*}{$\begin{array}{c}\text { SDS } \\
(2.5 \mathrm{hr}) \\
\text { \% yield }\end{array}$} & \multirow{2}{*}{$\begin{array}{l}\text { TX-100 } \\
(3.0 \mathrm{hr}) \\
\text { \% yield }\end{array}$} & \multirow{2}{*}{$\begin{array}{c}\text { M.P/B.P } \\
\left({ }^{\circ} \mathrm{C}\right)\end{array}$} \\
\hline 1 & 2 & & & & & \\
\hline \multirow{4}{*}{$\beta$-Naphthol } & $\mathrm{MeOH}$ & 89 & 93 & 90 & 90 & $70-71$ \\
\hline & $\mathrm{EtOH}$ & 90 & 93 & 90 & 90 & $35-37$ \\
\hline & 1-PrOH & 88 & 91 & 91 & 91 & $37-39$ \\
\hline & 2-PrOH & 91 & 94 & 91 & 91 & - \\
\hline \multirow{4}{*}{ 1-Bromonaphthalen-2-ol } & $\mathrm{MeOH}$ & 90 & 91 & 89 & 90 & $80-81$ \\
\hline & $\mathrm{EtOH}$ & 88 & 92 & 90 & 90 & $64-66$ \\
\hline & 1-PrOH & 88 & 96 & 90 & 89 & - \\
\hline & 2-PrOH & 91 & 94 & 89 & 89 & $37-39$ \\
\hline \multirow{4}{*}{ 1-Chloronaphthalen-2-ol } & $\mathrm{MeOH}$ & 90 & 90 & 93 & 89 & 64-65 \\
\hline & $\mathrm{EtOH}$ & 90 & 90 & 92 & 90 & $56-58$ \\
\hline & 1-PrOH & 91 & 91 & 91 & 88 & - \\
\hline & 2-PrOH & 91 & 91 & 94 & 91 & - \\
\hline \multirow{4}{*}{ 1-Iodonaphthalen-2-ol } & $\mathrm{MeOH}$ & 90 & 89 & 91 & 90 & $87-89$ \\
\hline & $\mathrm{EtOH}$ & 90 & 90 & 92 & 88 & $35-37$ \\
\hline & 1-PrOH & 89 & 90 & 96 & 88 & $37-39$ \\
\hline & 2-PrOH & 89 & 89 & 94 & 91 & - \\
\hline
\end{tabular}

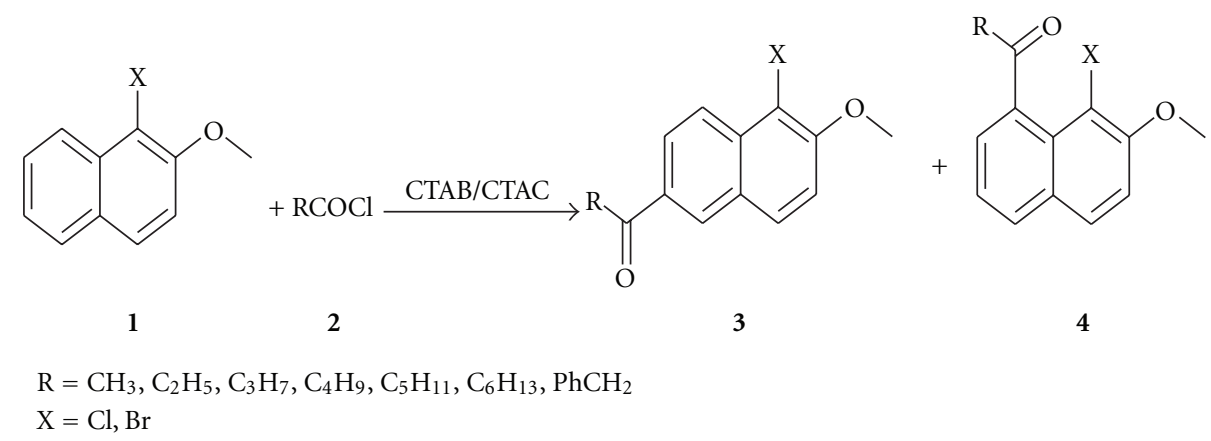

Scheme 2: Acylation of 1-halo-2-methoxy naphthalenes in micellar media.

presence of CTAB or CTAC, $(0.1 \mathrm{mmol}, 5 \mathrm{~mol} \%)$ were grounded with pestle for $2 \mathrm{hr}$ it gave 6-acyl-1-halo-2methoxynaphthalene(3) as the major product along with 8-acyl-1-halo-2-methoxy naphthalene(4) selectivity (98:02) represented as in Scheme 2, Table 4 and the reaction time versus conversion of 1-halo-2-methoxy naphthalenes using CTAB/CTAC (various amounts) as a catalyst were reported in Table 5.

Mortar-pestle grinding synthetic methods were to avoid solvent. The reaction times fairly reduced from $24 \mathrm{~h}$ to about 2-3 h (under Williamson's method to Mortar-pestle method) without many changes in the yield of products (Tables 1 and 2). Rate accelerations under grinding method could be attributed to bulk activation of molecules due to the conversion of mechanical energy (exerted due to grinding) into heat energy due to frictional forces operating between solid state reagents [1-3].
2.1. Calculation of Molecular Physicochemical Properties by Molinspiration. Drug likeness may be defined as a complex balance of various molecular properties and structure features which determine whether particular molecule is similar to the known drugs. These properties, mainly hydrophobicity, electronic distribution, hydrogen bonding characteristics, molecule size and flexibility, and various pharmacophoric features influence the behavior of molecule in a living organism, including bioavailability, transport properties, affinity to proteins, reactivity, toxicity, metabolic stability, and many others. Mol inspiration calculations are a set of simple molecular properties obtained by Lipinski Rule- 5 of $5 "$ [41, 42], which are useful to explore drug likeliness nature of a molecule. Lipinski's fifth rule states that a molecule possess "drug-likeliness" if the molecule has the properties: $\log P \leq 5$, molecular weight $\leq 500$, number of hydrogen bond acceptors $\leq 10$, and number of hydrogen bond donor's 
TABLE 2: Mortar-pestle-induced micellar mediated $O$-alkylation of hydroxy pyridines under solvent-free conditions.

\begin{tabular}{|c|c|c|c|c|}
\hline \multicolumn{2}{|l|}{ Reactants } & \multirow{2}{*}{$\begin{array}{l}\text { CTAB } \\
(1.5 \mathrm{hr}) \\
\text { \% yield }\end{array}$} & \multirow{2}{*}{$\begin{array}{c}\text { SDS } \\
(2.0 \mathrm{hr}) \\
\text { \% yield }\end{array}$} & \multirow{2}{*}{$\begin{array}{l}\text { TX-100 } \\
(2.5 \mathrm{hr}) \\
\% \text { yield }\end{array}$} \\
\hline 1 & 2 & & & \\
\hline \multirow{4}{*}{ 2-Hydroxy pyridine } & $\mathrm{MeOH}$ & 89 & 85 & 86 \\
\hline & $\mathrm{EtOH}$ & 90 & 87 & 87 \\
\hline & 1-PrOH & 90 & 86 & 82 \\
\hline & 2-PrOH & 90 & 87 & 89 \\
\hline \multirow{4}{*}{ 3-Hydrox pyridine } & $\mathrm{MeOH}$ & 91 & 85 & 82 \\
\hline & $\mathrm{EtOH}$ & 89 & 84 & 81 \\
\hline & 1-PrOH & 85 & 83 & 80 \\
\hline & 2-PrOH & 89 & 85 & 79 \\
\hline \multirow{4}{*}{ 4-Hydroxy pyridine } & $\mathrm{MeOH}$ & 91 & 88 & 81 \\
\hline & $\mathrm{EtOH}$ & 88 & 85 & 83 \\
\hline & 1-PrOH & 88 & 83 & 82 \\
\hline & 2-PrOH & 89 & 84 & 81 \\
\hline \multirow{4}{*}{ 5-Bromopyrimidin-2-ol } & $\mathrm{MeOH}$ & 91 & 87 & 89 \\
\hline & $\mathrm{EtOH}$ & 89 & 87 & 87 \\
\hline & 1-PrOH & 89 & 86 & 86 \\
\hline & 2-PrOH & 91 & 84 & 86 \\
\hline \multirow{4}{*}{ 6-Hydroxypicolinaldehyde } & $\mathrm{MeOH}$ & 92 & 88 & 89 \\
\hline & $\mathrm{EtOH}$ & 88 & 86 & 89 \\
\hline & 1-PrOH & 87 & 85 & 84 \\
\hline & 2-PrOH & 88 & 87 & 86 \\
\hline
\end{tabular}

$\leq 5$. Molecules violating more than one of these rules may have problems with bioavailability. For the prediction of oral bioavailability of drug molecules [43], lipophilicity ( $\log P$ value), and polar surface area (PSA) [44] values are the most important properties. The Calculated parameters from Mol inspiration methodology for each newly synthesized 6-acyl-1-halo-2-methoxynaphthalene derivative (entries 1 to 14) are compiled in Tables 6 and 7 and tested for specific activity with standard drugs Naproxen. For all the compounds, the calculated $\log P$ values were $\leq 5$ (in comparison with the accepted the upper limit) indicating the ability of the drug to penetrate through biomembranes and also good water solubility (according to Lipinski's rules). Further it is also interesting to note that all the synthesized molecules exhibit less than 140 PSA values indicating good intestinal absorption. Data presented in Table 7 are GPCR ligand activity, ion channel modulation, kinase inhibition activity, nuclear receptor ligand activity, Protease inhibitor, and Enzyme inhibitor are compared with Naproxen as standard drug indicate that most of the synthesized compounds depict by and large consistent negative values, which are in consonance with Lipinski's Rule -5 and are also comparable with Naproxen drug.

\section{Conclusions}

There are different shades of greener processes as we continue exploring several alternatives to conventional chemical transformations. That approach will require new environmentally benign syntheses of $O$-alkylation and acylation of naphthalene derivatives in aqueous micellar media. Thus, the present protocols show rate accelerations associated with high products yields, when compared with the similar reactions performed under classical conditions. Surfactants catalyze the reaction efficiently with short reaction times without using any harmful organic reagents and solvents. Molecular properties computed from Molinspiration methodology for all the synthesized 6-acyl-1-halo-2-methoxynaphthalene derivatives by and large indicate Drug likeliness behavior that is comparable to standard drugs. Thus, it is believed that the present work is a major breakthrough in the area of synthesis of 6-acyl, 1-halo-2-methoxy naphthalenes with potential biological activity.

\section{Experimental Details}

All reactions were followed by TLC with detection by UV light. Melting points were recorded on BUCHI B545 capillary melting point apparatus and are uncorrected. Infrared (IR) spectra were recorded on a Perkin Elmer FTIR spectrometer. ${ }^{1} \mathrm{H}$ and ${ }^{13} \mathrm{C}$ NMR spectra were recorded at a Varian VNMRS-400 and $100 \mathrm{MHz}$ spectrometer. The Samples were analyzed in $\mathrm{CDCl}_{3}, \mathrm{DMSO}-\mathrm{d}_{6}$ chemical shift values are reported in ppm relative to TMS as the internal reference. Mass spectra were recorded on a ZAB-HS mass spectrometer using ESI ionization. The isolation of pure products was carried out via preparative thin layer chromatography (silica gel $60 \mathrm{GF}_{254}$, Merck). Excess of solvent was evaporated under reduced pressure at a bath temperature of $50^{\circ} \mathrm{C}$. All solvents, organic and inorganic compounds, were purchased from Aldrich, Merck, Fluka, and Sdfine and used without further purification.

4.1. General Procedure for Etherification of $\beta$-Naphthols under Mortar Pestle Conditions. A mixture of substrate $(10 \mathrm{mmol})$, alcohol $(20 \mathrm{mmol})$, and micelles ( $1.0 \mathrm{mmol}, 25 \mathrm{~mol} \%)$ were added to a mortar. The mixture was grounded by mortar and pestle at room temperature, after the indicated reaction time, the reaction mixture was purified by thin layer chromatography (silicagel, EtOAc-petroleum ether, 1:9) to obtain the desired product.

4.2. General Procedure for Acylation of $\beta$-Naphthols under Mortar Pestle Conditions. 1-halo-2-methoxynaphthalene $(2 \mathrm{mmol})$ and acylchloride $(2 \mathrm{mmol})$ and CTAB $(0.1 \mathrm{mmol}$, $5 \mathrm{~mol} \%$ ) were added to a mortar and grinded with pestle till the reaction is completed as ascertained by TLC. The final products were isolated by absorbing the reaction mixture into silica gel and purifying it by column chromatography using ethyl acetate/hexane gradient. 
TABLE 3: Reaction of aromatic/heteroaromatic alcohol $(10 \mathrm{mmol})$ with methanol $(20 \mathrm{mmol})$ using micelles as catalysts.

\begin{tabular}{|c|c|c|c|c|c|c|}
\hline \multirow{2}{*}{ Aromatic/heteroaromatic alcohol (1) } & \multicolumn{2}{|c|}{ CTAB } & \multicolumn{2}{|c|}{ SDS } & \multicolumn{2}{|c|}{ TX-100 } \\
\hline & $\begin{array}{l}\text { Time } \\
(\mathrm{min})\end{array}$ & $\begin{array}{c}\text { Conversion of } 1 \\
(\%)\end{array}$ & $\begin{array}{l}\text { Time } \\
(\mathrm{min})\end{array}$ & $\begin{array}{c}\text { Conversion of } \mathbf{1} \\
(\%)\end{array}$ & $\begin{array}{l}\text { Time } \\
(\mathrm{min})\end{array}$ & $\begin{array}{c}\text { Conversion of } \mathbf{1} \\
(\%)\end{array}$ \\
\hline \multirow{4}{*}{$\beta$-Naphthol } & 60 & 50 & 60 & 50 & 60 & 45 \\
\hline & 80 & 63 & 90 & 65 & 100 & 60 \\
\hline & 100 & 75 & 120 & 75 & 140 & 78 \\
\hline & 120 & 93 & 150 & 90 & 180 & 90 \\
\hline \multirow{4}{*}{ 2-Hydroxy pyridine } & 60 & 60 & 60 & 55 & 60 & 50 \\
\hline & 70 & 73 & 90 & 68 & 90 & 65 \\
\hline & 80 & 81 & 120 & 77 & 120 & 77 \\
\hline & 90 & 89 & 120 & 85 & 150 & 86 \\
\hline
\end{tabular}

\subsection{Spectroscopic Analysis of Representative Compounds}

2-Methoxynaphthalene. ${ }^{1} \mathrm{H} \mathrm{NMR}\left(400 \mathrm{MHz}, \mathrm{CDCl}_{3}\right) \delta 3.91$ (s, 3H), 7.15-7.17 (d, $J=8.8 \mathrm{~Hz}, 1 \mathrm{H}), 7.29-7.36(\mathrm{~m}, 2 \mathrm{H})$, 7.43-7.47 (m, 1H), 7.72-7.77 (m, 3H); ${ }^{13} \mathrm{C}$ NMR $(50 \mathrm{MHz}$, $\left.\mathrm{CDCl}_{3}\right) \delta 55.6,105.9,113.7,124.4,126.2,127.8,128.2,129.1$, 129.9, 133.2, 153.8; IR (KBr, cm $\left.{ }^{-1}\right)$ 3067, 3008, 2963, 1632, 1599, 1477, 1462, 1452, 1440, 1398, 1391, 1368, 1262, 1218, $1197,1173,1152,1141,1118,1031,1017,963,948,874,838$, 818, 753, 743, 622, 481, 470; MS (EI) $m / z 159.2(\mathrm{M})^{+}$.

2-Ethoxynaphthalene. ${ }^{1} \mathrm{H} \mathrm{NMR}\left(400 \mathrm{MHz}, \mathrm{CDCl}_{3}\right) \delta 1.46(\mathrm{t}$, $J=7.2 \mathrm{~Hz}, 3 \mathrm{H}), 4.127(\mathrm{~d}, J=6.8 \mathrm{~Hz}, 2 \mathrm{H}), 7.14-7.15(\mathrm{~d}, J$ $=8.8 \mathrm{~Hz}, 1 \mathrm{H}), 7.28-7.39(\mathrm{~m}, 2 \mathrm{H}), 7.39-7.43(\mathrm{~m}, 1 \mathrm{H}), 7.69-$ $7.75(\mathrm{~m}, 3 \mathrm{H}) ;{ }^{13} \mathrm{C} \mathrm{NMR}\left(50 \mathrm{MHz}, \mathrm{CDCl}_{3}\right) \delta 14.8,64.1,106.5$, $118.9,123.4,126.2,126.7,128.2,129.1,129.2,133.6,156.8$; IR $\left(\mathrm{KBr}, \mathrm{cm}^{-1}\right)$ 3067, 2984, 2940, 2876, 1829, 1601, 1579, $1511,1457,1440,1395,1390,1367,1358,1350,1269,1259$, $1185,1166,1144,1122,1046,1020,958,928,873,823,762$, 719, 623, 477; MS (EI) $m / z 173.2(\mathrm{M})^{+}$.

2-Propoxynaphthalene. ${ }^{1} \mathrm{H}$ NMR $\left(400 \mathrm{MHz}, \mathrm{CDCl}_{3}\right) \delta 1.06$ $(\mathrm{t}, J=6.0 \mathrm{~Hz}, 3 \mathrm{H}), 1.83-1.86(\mathrm{~m}, 2 \mathrm{H}), 4.01(\mathrm{t}, 2 \mathrm{H}), 7.10-7.14$ (m, 2H), 7.30-7.41 (m, 2H), 7.69-7.74 (m, 3H); ${ }^{13} \mathrm{C}$ NMR $\left(50 \mathrm{MHz}, \mathrm{CDCl}_{3}\right) \delta 10.5,22.6,69.4,106.6,119.1,123.4$, 126.2, 126.6, 127.6, 128.8, 129.3, 134.6, 156.7; IR $\left(\mathrm{KBr}, \mathrm{cm}^{-1}\right)$ 3060, 2976, 2934, 2908, 2878, 1948, 1904, 1832, 1628, 1600, $1513,1479,1467,1448,1441,1392,1371,1357,1270,1260$, $1216,1146,1121,1046,1024,1017,1017,986,958,916,842$, $819,770,746,727,727,645,623,474$; MS (EI) $m / z 187.10$ $(\mathrm{M})^{+}$.

2-Isopropoxynaphthalene. ${ }^{1} \mathrm{H}$ NMR $\left(400 \mathrm{MHz}, \mathrm{CDCl}_{3}\right) \delta$ $1.45(\mathrm{~d}, J=6.0 \mathrm{~Hz}, 6 \mathrm{H}), 4.66-4.82(\mathrm{~m}, 1 \mathrm{H}), 7.13-7.18$ (m, 2H), 7.28-7.48 (m, 2H), 7.68-7.82 (m, 3H); ${ }^{13} \mathrm{C}$ NMR $\left(50 \mathrm{MHz}, \mathrm{CDCl}_{3}\right) \delta 22.0,69.8,108.4,119.7,123.4,126.2$, 126.6, 127.6, 128.8, 129.3, 134.6, 155.7; IR $\left(\mathrm{KBr}, \mathrm{cm}^{-1}\right) 3580$, 2981, 2936, 2876, 1948, 1904, 1832, 1628, 1600, 1581, 1510, $1468,1440,1388,1373,1356,1334,1216,1188,1171,1137$, $1118,1019,974,941,900,871,842,814,675,645,623,532$; MS (EI) $m / z 187.10(\mathrm{M})^{+}$.
1-Chloro-2-methoxynaphthalene. ${ }^{1} \mathrm{H} \quad \mathrm{NMR} \quad(400 \mathrm{MHz}$, $\left.\mathrm{CDCl}_{3}\right) \delta 4.03(\mathrm{~s}, 3 \mathrm{H}), 7.25(\mathrm{~d}, J=8.6 \mathrm{~Hz}, 1 \mathrm{H}), 7.37-7.52$ $(\mathrm{m}, 1 \mathrm{H}), 7.53-7.61(\mathrm{~m}, 1 \mathrm{H}), 7.79-7.69(\mathrm{~m}, 2 \mathrm{H}), 8.25(\mathrm{~d}$, $J=8.6 \mathrm{~Hz}, 1 \mathrm{H}) ;{ }^{13} \mathrm{C} \mathrm{NMR}\left(50 \mathrm{MHz}, \mathrm{CDCl}_{3}\right) \delta 57,105.9$, $113.7,124.4,126.2,127.8,128.2,129.1,129.9,133.2,153.8$; IR $\left(\mathrm{KBr}, \mathrm{cm}^{-1}\right)$ 3430, 3049, 2972, 2948, 2846, 2541, 1948, $1763,1625,1590,1505,1469,1355,1337,1273,1246,1187$, $1148,1068,1018,985,894,865,804,764,740,657,588,532$; MS (EI) $m / z 192(\mathrm{M})^{+}$.

1-Bromo-2-methoxynaphthalene. ${ }^{1} \mathrm{H} \quad \mathrm{NMR} \quad(400 \mathrm{MHz}$, $\left.\mathrm{CDCl}_{3}\right) \delta 4.04(\mathrm{~s}, 3 \mathrm{H}), 7.28(\mathrm{~d}, J=8.6 \mathrm{~Hz}, 1 \mathrm{H}), 7.39-7.53$ $(\mathrm{m}, 1 \mathrm{H}), 7.53-7.61(\mathrm{~m}, 1 \mathrm{H}), 7.76-7.89(\mathrm{~m}, 2 \mathrm{H}), 8.23(\mathrm{~d}, J$ $=8.6 \mathrm{~Hz}, 1 \mathrm{H}) ;{ }^{13} \mathrm{C} \mathrm{NMR}\left(50 \mathrm{MHz}, \mathrm{CDCl}_{3}\right) \delta 57.1,105.9$, $113.7,124.4,126.2,127.8,128.2,129.1,129.9,133.2,153.8$; IR $\left(\mathrm{KBr}, \mathrm{cm}^{-1}\right)$ 3430, 3045, 2970, 2941, 2841, 1620, 1594, 1500, 1466, 1454, 1351, 1334, 1270, 1245, 1185, 1153, 1134, $1061,1021,968,890,855,803,761,743,708,644,579$, $516 \mathrm{~cm}^{-1}$; MS (EI) $\mathrm{m} / z(\mathrm{M}-\mathrm{H})^{+} 236.10$.

1-Iodo-2-methoxynaphthalene. ${ }^{1} \mathrm{H} \mathrm{NMR}\left(400 \mathrm{MHz}, \mathrm{CDCl}_{3}\right)$ $\delta 4.03(\mathrm{~s}, 3 \mathrm{H}), 7.22(\mathrm{~d}, J=9.0 \mathrm{~Hz}, 1 \mathrm{H}), 7.34-7.42(\mathrm{~m}, 1 \mathrm{H})$, $7.50-7.56(\mathrm{~m}, 1 \mathrm{H}), 7.75(\mathrm{~d}, J=8.6 \mathrm{~Hz}, 1 \mathrm{H}), 7.84(\mathrm{~d}, J=$ $9.0 \mathrm{~Hz}, 1 \mathrm{H}), 8.15(\mathrm{~d}, J=8.6 \mathrm{~Hz}, 1 \mathrm{H}) ;{ }^{13} \mathrm{C} \mathrm{NMR}(50 \mathrm{MHz}$, $\left.\mathrm{CDCl}_{3}\right) \delta 57.3,87.8,113.0,124.4,128.2,128.3,130.4,131.3$, 130.0, 135.7, 156.7; IR (KBr, cm $\left.{ }^{-1}\right)$ 3042, 3006, 2969, 2937, 2838, 1617, 1587, 1551, 1497, 1451, 1423, 1346, 1328, 1263, 1242, 1181, 1153, 1132, 1058, 1021, 959, 887, 801, 761, 743; MS (EI) $m / z 285.06(\mathrm{M})^{+}$.

1-Bromo-2-ethoxynaphthalene. ${ }^{1} \mathrm{H} \mathrm{NMR}\left(400 \mathrm{MHz}, \mathrm{CDCl}_{3}\right)$ $\delta 1.38-1.41(\mathrm{t}, J=6.8 \mathrm{~Hz}, 3 \mathrm{H}), 4.13-4.14(\mathrm{q}, J=6.8 \mathrm{~Hz}, 2 \mathrm{H})$, 7.15-7.17 (d, $J=8.8 \mathrm{~Hz}, 1 \mathrm{H}), 7.29-7.36$ (m, 2H), 7.43-7.47 $(\mathrm{m}, 1 \mathrm{H}), 7.78-7.83(\mathrm{~m}, 3 \mathrm{H}) ;{ }^{13} \mathrm{C} \mathrm{NMR}\left(50 \mathrm{MHz}, \mathrm{CDCl}_{3}\right)$ $\delta$ 22.8, 64.6, 105.9, 113.7, 124.4, 126.2, 127.8, 128.2, 129.1, $129.9,133.2,153.8$.

1-Bromo-2-propoxynaphthalene. ${ }^{1} \mathrm{H} \quad \mathrm{NMR} \quad(400 \mathrm{MHz}$, $\left.\mathrm{CDCl}_{3}\right) \delta 1.03(\mathrm{t}, J=7.5 \mathrm{~Hz}, 3 \mathrm{H}), 1.80-1.86(\mathrm{~m}, 2 \mathrm{H}), 4.51$ $(\mathrm{t}, 7.5 \mathrm{~Hz}, 2 \mathrm{H}), 7.10-7.14(\mathrm{~m}, 2 \mathrm{H}), 7.30-7.41(\mathrm{~m}, 2 \mathrm{H})$, 7.69-7.74 (m, 3H); ${ }^{13} \mathrm{C} \mathrm{NMR}\left(50 \mathrm{MHz}, \mathrm{CDCl}_{3}\right) \delta 10.5,22.6$, 
TABLE 4: Reaction of 1-halo-2-methoxy naphthalene ( $2 \mathrm{mmol}$ ) with acylchlorides ( $2 \mathrm{mmol}$ ) using CTAB/CTAC as a catalyst.

Total
(\%) Yield \begin{tabular}{c}
$\begin{array}{c}\text { Products } \\
\text { ratio } \\
(\mathbf{3}: \mathbf{4})\end{array}$ \\
\hline $\mathbf{2 0}$
\end{tabular}

Duration of all reactions was $2 \mathrm{hr}$.

$78.4,113.6,123.4,126.2,126.6,127.6,128.8,129.3,131.3$, 132.6, 154.7; MS (EI) $m / z 266.04(\mathrm{M})^{+}$.

1-Bromo-2-isopropoxy Naphthalene. ${ }^{1} \mathrm{H}$ NMR $(400 \mathrm{MHz}$, $\left.\mathrm{CDCl}_{3}\right) \delta 1.45(\mathrm{~d}, J=6.0 \mathrm{~Hz}, 6 \mathrm{H}), 4.61-4.87(\mathrm{~m}, 1 \mathrm{H}), 7.28(\mathrm{~d}$, $J=8.6 \mathrm{~Hz}, 1 \mathrm{H}), 7.39-7.53(\mathrm{~m}, 1 \mathrm{H}), 7.53-7.61$ (m, 1H), 7.76$7.85(\mathrm{~m}, 2 \mathrm{H}), 8.23(\mathrm{~d}, J=8.6 \mathrm{~Hz}, 1 \mathrm{H}) ;{ }^{13} \mathrm{C} \mathrm{NMR}(50 \mathrm{MHz}$, $\left.\mathrm{CDCl}_{3}\right) \delta 22.0,69.8,108.4,119.7,123.4,126.2,126.6,127.6$, 128.8, 129.3, 134.6, 155.7; IR $\left(\mathrm{KBr}, \mathrm{cm}^{-1}\right) 3580,2981,2936$, 2876, 1948, 1904, 1832, 1628, 1600, 1581, 1510, 1468, 1440, $1388,1373,1356,1334,1216,1188,1171,1137,1118,1019$,
TABLE 5: Reaction of 1-halo-2-methoxynaphthalene (2 mmol) With $2 \mathbf{g}(2 \mathrm{mmol})$ using CTAB/CTAC (various amounts) as a catalyst.

\begin{tabular}{|c|c|c|c|}
\hline \multicolumn{2}{|c|}{ Naphthalene CTAB/CTAC(mmol) } & \multirow{2}{*}{$\begin{array}{c}\text { Time } \\
(\mathrm{min})\end{array}$} & \multirow{2}{*}{$\begin{array}{c}\begin{array}{c}\text { Conversion of } \mathbf{1} \\
(\%)\end{array} \\
70\end{array}$} \\
\hline \multirow{4}{*}{$1 \mathrm{a}$} & \multirow{4}{*}{0.02} & & \\
\hline & & 80 & 73 \\
\hline & & 100 & 75 \\
\hline & & 120 & 80 \\
\hline \multirow{4}{*}{$1 \mathbf{a}$} & \multirow{4}{*}{0.04} & 60 & 72 \\
\hline & & 80 & 78 \\
\hline & & 100 & 82 \\
\hline & & 120 & 85 \\
\hline \multirow{4}{*}{$1 \mathrm{a}$} & \multirow{4}{*}{0.06} & 60 & 74 \\
\hline & & 80 & 80 \\
\hline & & 100 & 84 \\
\hline & & 120 & 86 \\
\hline \multirow{4}{*}{ 1a } & \multirow{4}{*}{0.08} & 60 & 76 \\
\hline & & 80 & 83 \\
\hline & & 100 & 86 \\
\hline & & 120 & 90 \\
\hline \multirow{4}{*}{$1 \mathbf{a}$} & \multirow{4}{*}{0.10} & 60 & 80 \\
\hline & & 80 & 85 \\
\hline & & 100 & 89 \\
\hline & & 120 & 93 \\
\hline \multirow{4}{*}{$1 b$} & \multirow{4}{*}{0.02} & 60 & 70 \\
\hline & & 80 & 75 \\
\hline & & 100 & 78 \\
\hline & & 120 & 83 \\
\hline \multirow{4}{*}{$1 b$} & \multirow{4}{*}{0.04} & 60 & 72 \\
\hline & & 80 & 75 \\
\hline & & 100 & 79 \\
\hline & & 120 & 85 \\
\hline \multirow{4}{*}{$1 b$} & \multirow{4}{*}{0.06} & 60 & 74 \\
\hline & & 80 & 79 \\
\hline & & 100 & 80 \\
\hline & & 120 & 87 \\
\hline \multirow{4}{*}{$1 b$} & \multirow{4}{*}{0.08} & 60 & 76 \\
\hline & & 80 & 81 \\
\hline & & 100 & 83 \\
\hline & & 120 & 89 \\
\hline \multirow{4}{*}{$1 b$} & \multirow{4}{*}{0.10} & 60 & 80 \\
\hline & & 80 & 85 \\
\hline & & 100 & 88 \\
\hline & & 120 & 93 \\
\hline
\end{tabular}

974, 941, 900, 871, 842, 814, 675, 645, 623, 532; MS (EI) $m / z$ $266.10(\mathrm{M})^{+}$.

4-Methoxy Pyridine. ${ }^{1} \mathrm{H} \mathrm{NMR}\left(400 \mathrm{MHz}, \mathrm{CDCl}_{3}\right) \delta 3.84(\mathrm{~s}$, $3 \mathrm{H}), 6.81(\mathrm{~d}, J=8.6 \mathrm{~Hz}, 2 \mathrm{H}), 8.43(\mathrm{~d}, J=8.6 \mathrm{~Hz}, 1 \mathrm{H}) ;{ }^{13} \mathrm{C}$ 
TABLE 6: Molinspiration calculations of compounds.

\begin{tabular}{|c|c|c|c|c|c|c|c|}
\hline S. number & 6-Acyl-1-halo-2-methoxynaphthalene & M.Wt & $\operatorname{miLog} P$ & TPSA & $\mathrm{OH}-\mathrm{NH}$ & $n$-Vio & Volume \\
\hline 1 & 1-(1-Bromo-2-methoxynaphthalen-6-yl)ethanone & 279.13 & 3.789 & 26.305 & 0 & 2 & 0 \\
\hline 2 & 1-(1-Bromo-2-methoxynaphthalen-6-yl)pentan-1-one & 321.21 & 5.356 & 26.305 & 0 & 2 & 1 \\
\hline 3 & 1-(1-Bromo-2-methoxynaphthalen-6-yl)hexan-1-one & 335.24 & 5.861 & 26.305 & 0 & 2 & 1 \\
\hline 4 & 1-(1-Bromo-2-methoxynaphthalen-6-yl)-4-methylpentan-1-one & 335.24 & 5.337 & 26.305 & 0 & 2 & 1 \\
\hline 5 & 1-(1-Bromo-2-methoxynaphthalen-6-yl)-5-methylhexan-1-one & 349.26 & 5.842 & 26.305 & 0 & 2 & 1 \\
\hline 6 & 1-(1-Bromo-2-methoxynaphthalen-6-yl)-3,3-dimethylbutan-1-one & 335.24 & 5.386 & 26.305 & 0 & 2 & 1 \\
\hline 7 & 1-(1-Bromo-2-methoxynaphthalen-6-yl)-2-phenylethanone & 355.23 & 5.217 & 26.305 & 0 & 2 & 1 \\
\hline 8 & 1-(1-Chloro-2-methoxynaphthalen-6-yl)ethanone & 234.68 & 3.658 & 26.305 & 0 & 2 & 0 \\
\hline 9 & 1-(1-Chloro-2-methoxynaphthalen-6-yl)pentan-1-one & 276.76 & 5.225 & 26.305 & 0 & 2 & 1 \\
\hline 10 & 1-(1-Chloro-2-methoxynaphthalen-6-yl)hexan-1-one & 290.79 & 5.73 & 26.305 & 0 & 2 & 1 \\
\hline 11 & 1-(1-Chloro-2-methoxynaphthalen-6-yl)-4-methylpentan-1-one & 290.79 & 5.206 & 26.305 & 0 & 2 & 1 \\
\hline 12 & 1-(1-Chloro-2-methoxynaphthalen-6-yl)-5-methylhexan-1-one & 304.81 & 5.711 & 26.305 & 0 & 2 & 1 \\
\hline 13 & 1-(1-Chloro-2-methoxynaphthalen-6-yl)-3,3-dimethylbutan-1-one & 290.79 & 5.255 & 26.305 & 0 & 2 & 1 \\
\hline 14 & 1-(1-Chloro-2-methoxynaphthalen-6-yl)-2-phenylethanone & 310.78 & 5.086 & 26.305 & 0 & 2 & 1 \\
\hline STD & Naproxen & 264.70 & 3.983 & 46.533 & 1 & 3 & 0 \\
\hline
\end{tabular}

TABLE 7: Drug likeness of compounds.

\begin{tabular}{|c|c|c|c|c|c|}
\hline S. number & 6-Acyl-1-halo-2-methoxynaphthalene & GPCRL & ICM & KI & NRL \\
\hline 1 & 1-(1-Bromo-2-methoxynaphthalen-6-yl)ethanone & -0.56 & -0.51 & -0.71 & -0.57 \\
\hline 2 & 1-(1-Bromo-2-methoxynaphthalen-6-yl)pentan-1-one & -0.18 & -0.36 & -0.42 & -0.20 \\
\hline 3 & 1-(1-Bromo-2-methoxynaphthalen-6-yl)hexan-1-one & -0.13 & -0.34 & -0.35 & -0.14 \\
\hline 4 & 1-(1-Bromo-2-methoxynaphthalen-6-yl)-4-methylpentan-1-one & -0.14 & -0.33 & -0.39 & -0.14 \\
\hline 5 & 1-(1-Bromo-2-methoxynaphthalen-6-yl)-5-methylhexan-1-one & -0.09 & -0.31 & -0.34 & -0.08 \\
\hline 6 & 1-(1-Bromo-2-methoxynaphthalen-6-yl)-3,3-dimethylbutan-1-one & -0.19 & -0.27 & -0.46 & -0.32 \\
\hline 7 & 1-(1-Bromo-2-methoxynaphthalen-6-yl)-2-phenylethanone & -0.06 & -0.30 & -0.24 & -0.05 \\
\hline 8 & 1-(1-Chloro-2-methoxynaphthalen-6-yl)ethanone & -0.50 & -0.22 & -0.67 & -0.49 \\
\hline 9 & 1-(1-Chloro-2-methoxynaphthalen-6-yl)pentan-1-one & -0.13 & -0.12 & -0.39 & -0.14 \\
\hline 10 & 1-(1-Chloro-2-methoxynaphthalen-6-yl)hexan-1-one & -0.08 & -0.11 & -0.32 & -0.08 \\
\hline 11 & 1-(1-Chloro-2-methoxynaphthalen-6-yl)-4-methylpentan-1-one & -0.10 & -0.10 & -0.37 & -0.08 \\
\hline 12 & 1-(1-Chloro-2-methoxynaphthalen-6-yl)-5-methylhexan-1-one & -0.04 & -0.09 & -0.31 & -0.02 \\
\hline 13 & 1-(1-Chloro-2-methoxynaphthalen-6-yl)-3,3-dimethylbutan-1-one & -0.14 & -0.04 & -0.43 & -0.26 \\
\hline 14 & 1-(1-Chloro-2-methoxynaphthalen-6-yl)-2-phenylethanone & -0.02 & -0.10 & -0.21 & 0.00 \\
\hline STD & Naproxen & -0.06 & 0.00 & -0.36 & 0.18 \\
\hline
\end{tabular}

GPCR: ligand, ICM: Ion channel modulator, KI: Kinase inhibitor, NRL: Nuclear receptor ligand.

$\mathrm{NMR}\left(50 \mathrm{MHz}, \mathrm{CDCl}_{3}\right) \delta 55.1,109.9,151.4,165.4, \mathrm{MS}(\mathrm{EI})$ $\mathrm{m} / \mathrm{z} 110.3(\mathrm{M})^{+}$, (B.P. $\left.168.2^{\circ} \mathrm{C}\right)$.

3-Methoxy Pyridine. ${ }^{1} \mathrm{H}$ NMR (400 MHz, $\left.\mathrm{CDCl}_{3}\right) \delta 3.84(\mathrm{~s}$, $3 \mathrm{H}), 7.34(\mathrm{t}, J=8.65, J=4.75 \mathrm{~Hz}, 1 \mathrm{H}), 7.38(\mathrm{~d}, J=8.65 \mathrm{~Hz}$, $1 \mathrm{H}) ; 8.19(\mathrm{~d}, J=4.75 \mathrm{~Hz}, 1 \mathrm{H}) ; 8.32(\mathrm{~s}, 1 \mathrm{H}) ; \mathrm{MS}(\mathrm{EI}) \mathrm{m} / z$ $110.3(\mathrm{M})^{+},\left(\right.$B.P. $\left.168.4^{\circ} \mathrm{C}\right)$.

2-Methoxy Pyridine. ${ }^{1} \mathrm{H} \mathrm{NMR}\left(400 \mathrm{MHz}, \mathrm{CDCl}_{3}\right) \delta 3.94(\mathrm{~s}$, $3 \mathrm{H}), 6.72(\mathrm{~d}, J=8.65 \mathrm{~Hz}, 1 \mathrm{H}) ; 6.82(\mathrm{t}, J=8.65, J=4.75 \mathrm{~Hz}$, $1 \mathrm{H}), 7.59(\mathrm{t}, J=8.65, J=4.75 \mathrm{~Hz} 1 \mathrm{H}) ; 8.18(\mathrm{~d}, J=4.75 \mathrm{~Hz}$, $1 \mathrm{H}) ;{ }^{13} \mathrm{C} \mathrm{NMR}\left(50 \mathrm{MHz}, \mathrm{CDCl}_{3}\right) \delta 53.1,110.9,116.4,138$, 147, 165.4, MS (EI) $m / z 110.3(\mathrm{M})^{+}$, (B.P. $\left.142.5^{\circ} \mathrm{C}\right)$.
4-Ethoxy Pyridine. ${ }^{1} \mathrm{H}$ NMR $\left(400 \mathrm{MHz}, \mathrm{CDCl}_{3}\right) \delta 1.34(\mathrm{q}$, $2 \mathrm{H}), 3.94(\mathrm{t}, 3 \mathrm{H}), 6.81(\mathrm{~d}, J=8.4 \mathrm{~Hz}, 2 \mathrm{H}), 8.43(\mathrm{~d}, J=8.4 \mathrm{~Hz}$, $2 \mathrm{H})$; MS (EI) $m / z 123.8(\mathrm{M})^{+}$, (B.P. $\left.165^{\circ} \mathrm{C}\right)$.

6-Methoxypicolinaldehyde. ${ }^{1} \mathrm{H}$ NMR (400 MHz, DMSO) $\delta$ $3.971(\mathrm{~s}, 3 \mathrm{H}), 6.986(\mathrm{~d}, J=8.3 \mathrm{~Hz}, 1 \mathrm{H}), 8.108-8.136(\mathrm{~m}$, $1 \mathrm{H}), 8.770$ (d, $J=2.5 \mathrm{~Hz}, 1 \mathrm{H}) ; 9.968$ (s, 1H); MS (EI) $m / z$ $137.9(\mathrm{M})^{+}$.

5-Bromo-2-methoxypyrimidine. ${ }^{1} \mathrm{H} \quad \mathrm{NMR} \quad(400 \mathrm{MHz}$, DMSO) $\delta 3.915(\mathrm{~s}, 3 \mathrm{H}), 8.765(\mathrm{~s}, 2 \mathrm{H})$; MS (EI) $\mathrm{m} / z$ $188.9(\mathrm{M})^{+}$.

1-(5-Bromo-6-methoxynaphthalen-2-yl)hexan-1-one. ${ }^{1} \mathrm{H}$ $\mathrm{NMR}\left(400 \mathrm{MHz}, \mathrm{CDCl}_{3}\right) \delta 0.95(\mathrm{t}, J=6.6 \mathrm{~Hz}, 3 \mathrm{H})$, 
$1.38-1.42(\mathrm{~m}, 4 \mathrm{H}), 1.93(\mathrm{~d}, J=7.7 \mathrm{~Hz}, 2 \mathrm{H}), 3.03(\mathrm{t}, J=$ $7.7 \mathrm{~Hz}, 2 \mathrm{H}), 4.02(\mathrm{~s}, 3 \mathrm{H}), 7.28(\mathrm{~d}, J=8.7 \mathrm{~Hz}, 1 \mathrm{H}), 7.98(\mathrm{~d}$, $J=8.7 \mathrm{~Hz}, 1 \mathrm{H}), 8.04(\mathrm{~s}, 1 \mathrm{H}), 8.23(\mathrm{~d}, J=8.7 \mathrm{~Hz}, 1 \mathrm{H}), 8.41$ (d, $J=8.7 \mathrm{~Hz}, 1 \mathrm{H})$; IR (KBr) 3430, 3057, 3016, 2957, 2922, 2882, 2126, 1943, 1716, 1672, 1621, 1559, 1492, 1472, 1440, $1408,1357,1328,1272,1252,1219,1177,1065,965,908$, $863,822,801,768,732,663,596,520,467 \mathrm{~cm}^{-1}$; MS (ES) $\mathrm{m} / \mathrm{z}(337.0 \mathrm{M})^{+2}$.

\section{Acknowledgments}

The authors are thankful to Professor P. K. Saiprakash for constant encouragement and Heads of the Chemistry Department at Osmania University and Nizam College, Hyderabad for providing facilities.

\section{References}

[1] N. B. Singh, R. J. Singh, and N. P. Singh, "Organic solid state reactivity," Tetrahedron, vol. 50, no. 22, pp. 6441-6493, 1994.

[2] G. R. Desiraju, "Reactivity of organic solids," Solid State Ionics, vol. 101-103, no. 1, pp. 839-842, 1997.

[3] K. Tanaka and F. Toda, "Solvent-free organic synthesis," Chemical Reviews, vol. 100, no. 3, pp. 1025-1074, 2000.

[4] R. A. Sheldon, "Green solvents for sustainable organic synthesis: state of the art," Green Chemistry, vol. 7, no. 5, pp. 267-278, 2005.

[5] A. L. Garay, A. Pichon, and S. L. James, "Solvent-free synthesis of metal complexes," Chemical Society Reviews, vol. 36, no. 6, pp. 846-855, 2007.

[6] A. Orita, L. Jiang, T. Nakano, N. Ma, and J. Otera, "Solventless reaction dramatically accelerates supramolecular selfassembly," Chemical Communications, no. 13, pp. 1362-1363, 2002.

[7] F. Toda, K. Tanaka, and S. Iwata, "Oxidative coupling reactions of phenols with $\mathrm{FeCl} 3$ in the solid state," Journal of Organic Chemistry, vol. 54, no. 13, pp. 3007-3009, 1989.

[8] F. Toda, K. Kiyoshige, and M. Yagi, " $\mathrm{NaBH}_{4}$ reduction of ketones in the solid state," Angewandte Chemie International Edition in English, vol. 28, pp. 320-321, 1989.

[9] V. P. Balema, J. W. Wiench, M. Pruski, and V. K. Pecharsky, "Mechanically induced solid-state generation of phosphorus ylides and the solvent-free wittig reaction," Journal of the American Chemical Society, vol. 124, no. 22, pp. 6244-6245, 2002.

[10] J. M. Harrowfield, R. J. Hart, and C. R. Whitaker, "Magnesium and aromatics: mechanically-induced grignard and McMurry reactions," Australian Journal of Chemistry, vol. 54, no. 7, pp. 423-425, 2001.

[11] Z. X. Wang and L. Q. Hue, "Solventless syntheses of pyrazole derivatives," Green Chemistry, vol. 6, no. 2, pp. 90-92, 2004.

[12] V. M. Markhele, S. A. Sadaphal, and M. S. Shingare, "An efficient one-pot synthesis of polyhydroquinolines at room temperature using MCM-41 catalyst under solvent-free conditions," Bulletin of the Catalysis Society of India, vol. 6, pp. 125-131, 2007.

[13] K. C. Rajanna, K. Ramesh, S. Ramgopal, S. Shylaja, P. G. Reddy, and P. K. Saiprakash, "Poly ethylene glycols as efficient media for the synthesis of $\beta$-nitro styrenes from $\alpha, \beta$-unsaturated carboxylic acids and metal nitrates under conventional and non-conventional conditions," Green and Sustainable Chemistry, vol. 1, pp. 132-148, 2011.
[14] S. Ramgopal, K. Ramesh, A. Chakradhar, N. M. Reddy, and K. C. Rajanna, "Metal nitrate driven nitro Hunsdiecker reaction with $\alpha, \beta$-unsaturated carboxylic acids under solventfree conditions," Tetrahedron Letters, vol. 48, no. 23, pp. 40434045, 2007.

[15] A. Chakradhar, R. Roopa, K. C. Rajanna, and P. K. Saiprakash, "Vilsmeier-haack bromination of aromatic compounds with $\mathrm{KBr}$ and N-bromosuccinimide under solvent-free conditions," Synthetic Communications, vol. 39, no. 10, pp. 1817-1824, 2009.

[16] M. Venkateswarlu, K. C. Rajanna, M. S. Kumar, U. U. Kumar, S. Ramgopal, and P. K. Saiprakash, "Rate enhancements in the acetlation and benzoylation of certain aromatic compounds with vilsmeier-haack reagents using acetamide, benzamide and oxychlorides under non- conventional conditions," International Journal of Organic Chemistry, vol. 1, pp. 233-241, 2011.

[17] M. Pagliaro, R. Ciriminna, H. Kimura, M. Rossi, and C. D. Pina, "From glycerol to value-added products," Angewandte Chemie International Edition, vol. 46, no. 24, pp. 4434-4440, 2007.

[18] N. Baggett, Comprehensive Organic Chemistry, vol. 1 of Edited by D. Barton, W. D. Ollis and J. F. Stoddart, Pergaman, Oxford, UK, 1979.

[19] J. March, Advanced Organic Chemistry, Reactions, Mechanism and Structure, John Wiley \& Sons, New York, NY, USA, 4th edition, 1992.

[20] S. Kim, K. N. Chung, and S. Yang, "Direct synthesis of ethers via zinc chloride mediated etherification of alcohols in dichloroethane," Journal of Organic Chemistry, vol. 52, no. 17, pp. 3917-3919, 1987.

[21] L. Karas and W. J. Piel, Kirk-Othmer Encyclopedia of Chemical Technology, vol. 9, John Wiley \& Sons, New York, NY, USA, 4th edn edition, 1992.

[22] P. Salehi, N. Iranpoor, and F. K. Behbahani, "Selective and efficient alcoholyses of allylic, secondary- and tertiary benzylic alcohols in the presence of iron(III)," Tetrahedron, vol. 54, no. 5-6, pp. 943-948, 1998.

[23] J. Pozniczek, A. Micek-llnicka, A. Lubanska, and A. Bielanski, "Catalytic synthesis of ethyl-tert-butyl ether on Dawson type heteropolyacid," Applied Catalysis A, vol. 286, pp. 52-60, 2005.

[24] T. Ollevier and T. M. Mwene-Mbeja, "Bismuth triflate catalyzed Claisen rearrangement of allyl naphthyl ethers," Tetrahedron Letters, vol. 47, no. 24, pp. 4051-4055, 2006.

[25] K. C. K. Swamy, N. N. B. Kumar, E. Balaraman, and K. V. P. P. Kumar, "Mitsunobu and related eactions: advances and applications," Chemical Reviews, vol. 109, pp. 551-2651, 2009.

[26] M. Debabrata and S. L. Buchwald, "Cu-catalyzed arylation of phenols: synthesis of sterically hindered and heteroaryl diaryl ethers," Journal of Organic Chemistry, vol. 75, no. 5, pp. 17911794, 2010.

[27] M. D. Maiti, "Chemoselectivity in the Cu-catalyzed $O$ arylation of phenols and aliphatic alcohols," Chemical Communications, vol. 47, no. 29, pp. 8340-8342, 2011.

[28] G. A. Olah, Friedel-Crafts Chemistry, Wiley-Interscience, New York, NY, USA, 1973.

[29] T. Yamato, C. Hideshima, G. K. S. Prakash, and G. A. Olah, "Organic reactions catalyzed by solid superacids. 5. Perfluorinated sulfonic acid resin (Nafion-H) catalyzed intramolecular Friedel-Crafts acylation," Journal of Organic Chemistry, vol. 56, no. 12, pp. 3955-3957, 1991.

[30] T. Mukaiyama, T. Ohno, T. Nishimura, and S. Suda, "The catalytic friedel-crafts acylation reaction using a catalyst 
generated from $\mathrm{GaCl} 3$ and a silver salt," Chemistry Letters, pp. 1059-1062, 1991.

[31] T. Mukaiyama, K. Suzuki, J. S. Han, and S. Kobayashi, "A novel catalyst system, antimony $(\mathrm{V})$ chloride-lithium perchlorate (SbCl5-LiClO4), in the friedel-crafts acylation reaction," Chemistry Letters, pp. 435-438, 1992.

[32] A. Kawada, S. Mitamura, and S. Kobayashi, "Lanthanide trifluoromethanesulfonates as reusable catalysts: catalytic FriedelCrafts acylation," Journal of the Chemical Society, Chemical Communications, no. 14, pp. 1157-1158, 1993.

[33] P. A. Evans, J. D. Nelson, and A. L. Stanley, "Directed lithiation/transmetalation approach to palladium-catalyzed crosscoupling acylation reactions," Journal of Organic Chemistry, vol. 60, no. 7, pp. 2298-2301, 1995.

[34] S. H. Kim, M. V. Hanson, and R. D. Rieke, "Direct formation of organomanganese bromides using Rieke manganese," Tetrahedron Letters, vol. 37, no. 13, pp. 2197-2200, 1996.

[35] J. H. Fendler and E. J. Fendler, Catalysis in Micellar and Macromolecular Systems, Academic Press, London, UK, 1975.

[36] F. M. Menger, J. U. Rhee, and H. K. Rhee, "Applications of surfactants to synthetic organic chemistry," Journal of Organic Chemistry, vol. 40, no. 25, pp. 3803-3805, 1975.

[37] P. T. Anastas and J. C. Warner, Green Chemistry: Theory and Practice, Oxford University Press, New York, NY, USA, 1998.

[38] L. M. Wang, N. Jiao, J. Qiu et al., "Sodium stearatecatalyzed multicomponent reactions for efficient synthesis of spirooxindoles in aqueous micellar media," Tetrahedron, vol. 66, no. 1, pp. 339-343, 2010.

[39] Y. Watanabe, K. Sawada, and M. Hayashi, "A green method for the self-aldol condensation of aldehydes using lysine," Green Chemistry, vol. 12, no. 3, pp. 384-386, 2010.

[40] H. Firouzabadi, N. Iranpoor, S. Kazemi, A. Ghaderi, and A. Garzan, "Highly efficient halogenation of organic compounds with halides catalyzed by cerium(III) chloride heptahydrate using hydrogen peroxide as the terminal oxidant in water," Advanced Synthesis and Catalysis, vol. 351, no. 11-12, pp. 1925-1932, 2009.

[41] http://www.molinspiration.com.

[42] C. A. Lipinski, F. Lombardo, B. W. Dominy, and P. J. Feeney, "Experimental and computational approaches to estimate solubility and permeability in drug discovery and development settings," Advanced Drug Delivery Reviews, vol. 23, no. 1-3, pp. 3-25, 1997.

[43] D. F. Veber, S. R. Johnson, H. Y. Cheng, B. R. Smith, K. W. Ward, and K. D. Kopple, "Molecular properties that influence the oral bioavailability of drug candidates," Journal of Medicinal Chemistry, vol. 45, no. 12, pp. 2615-2623, 2002.

[44] P. Ertl, B. Rohde, and P. Selzer, "Fast calculation of molecular polar surface area as a sum of fragment-based contributions and its application to the prediction of drug transport properties," Journal of Medicinal Chemistry, vol. 43, no. 20, pp. 3714-3717, 2000. 


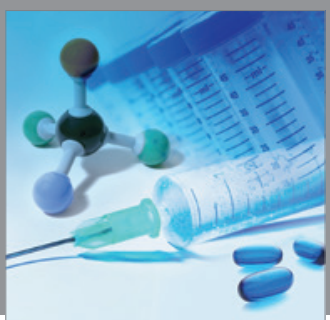

International Journal of

Medicinal Chemistry



Carbohydrate Chemistry

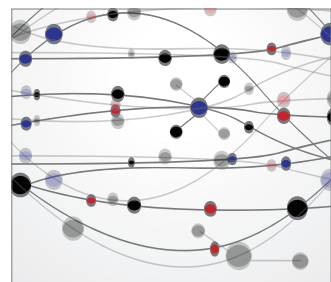

The Scientific World Journal
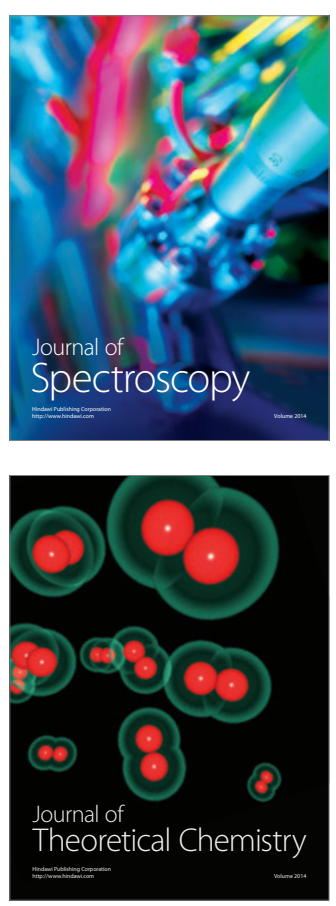
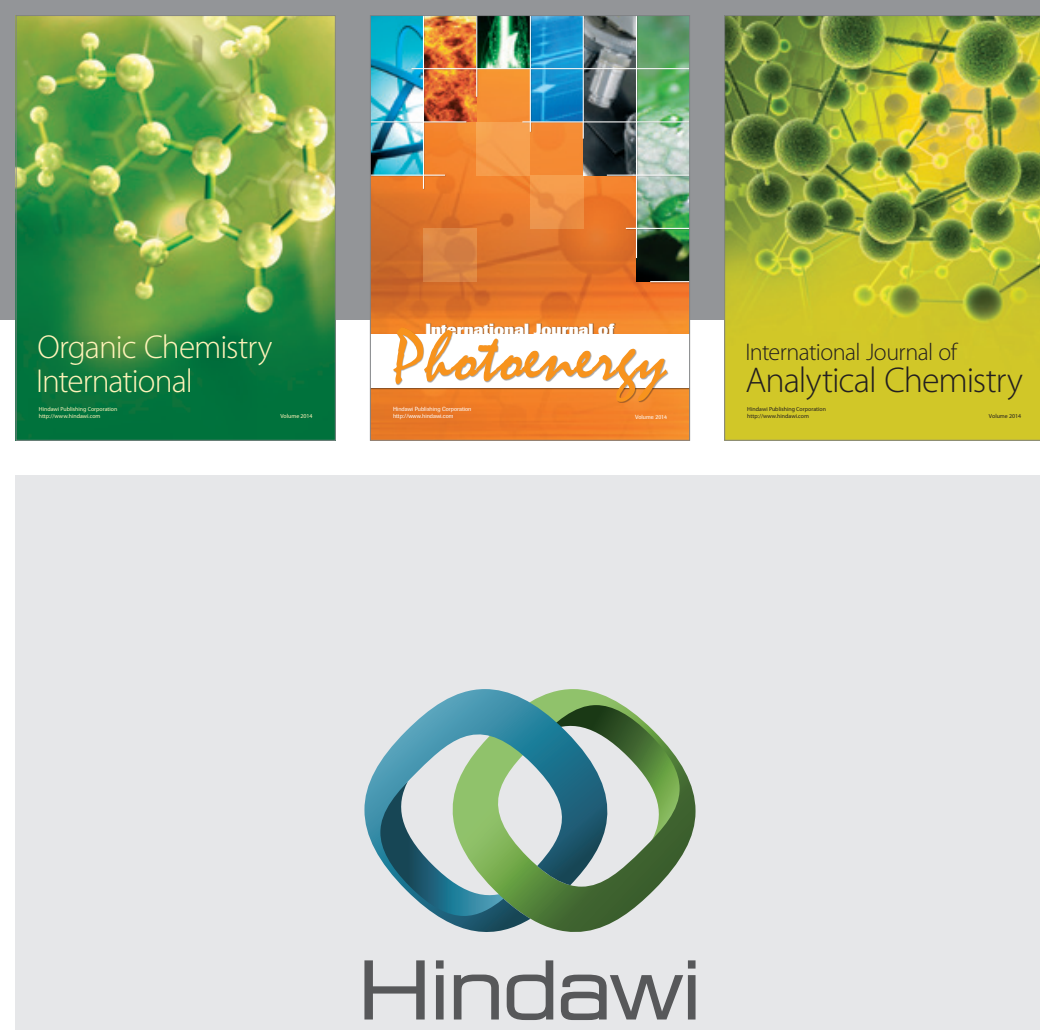

Submit your manuscripts at

http://www.hindawi.com
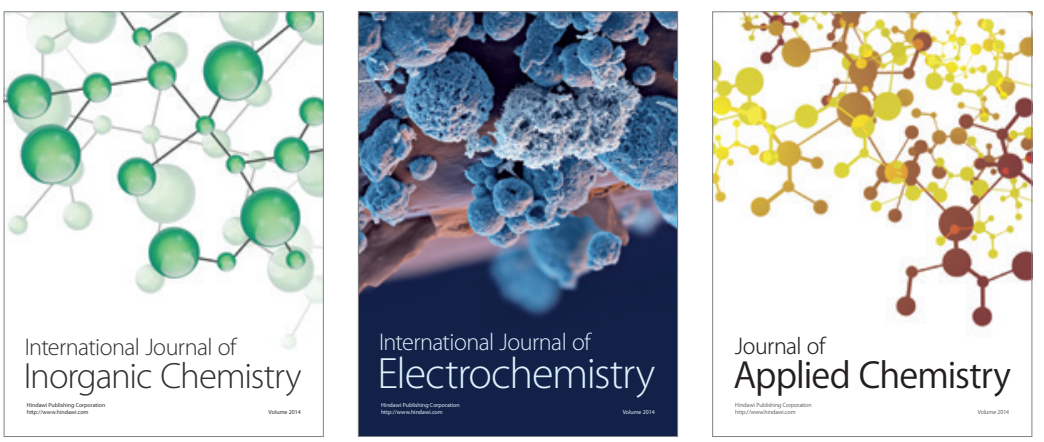

Journal of

Applied Chemistry
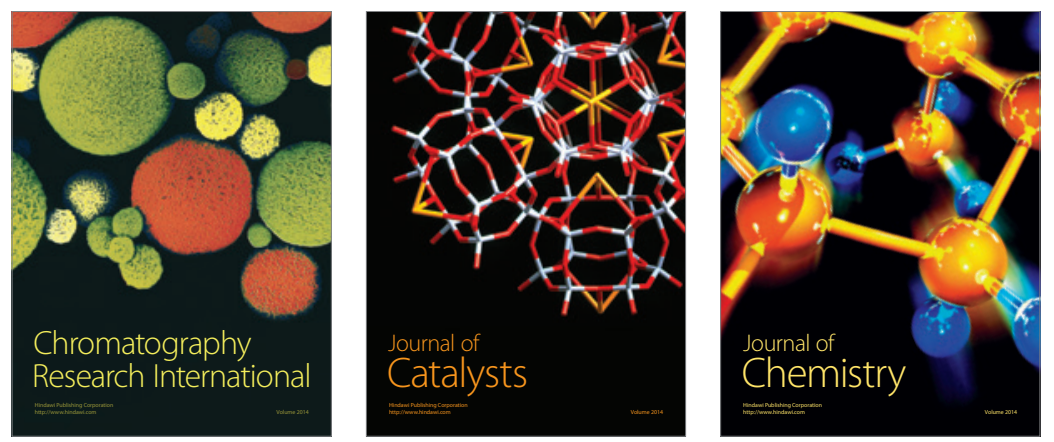
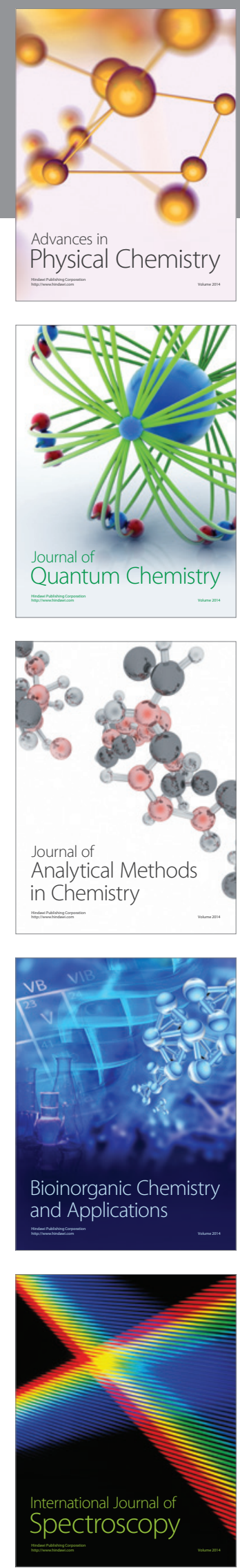